\title{
Improved the Monitoring Performance of Oil Pipeline Using Underwater Wireless Sensor Network
}

\author{
Wassim M.Jassim*, A.E.Abdelkareem \\ Dep. of Networks Eng., Al-Nahrain University, Baghdad, IRAQ
}

*Corresponding Author: Wassim M.Jassim, Dep. of Networks Eng., Al-Nahrain University, Baghdad, IRAQ

\begin{abstract}
In the last two decades, underwater acoustic sensor networks have begun to be used for commercial and non-commercial purposes. In this paper, the focus will be on improving the monitoring performance system of oil pipelines. Linear wireless sensor networks are a model of underwater applications for which many solutions have been developed through several research studies in previous years for data collection research. In underwater environments, there are certain inherent limitations, like large propagation delays, high error rate, limited bandwidth capacity, and communication with short-range. Many deployment algorithms and routing algorithms have been used in this field. In this work a new hierarchical network model proposed by mean adding new nodes to the parents/ child relationship of the hierarchal linear structure with improvement to Smart Redirect or Jump algorithm (SRJ) which supports this type of network in underwater. This improved algorithm is used in an underwater linear wireless sensor network for data transfer to reduce the complexity in routing algorithms for relay nodes andminimizeoverall delay in network communication. This work is implemented using OMNET++ and MATLAB based on their integration. The results obtained based on throughput, energy consumption, and end to the end delay
\end{abstract}

.Keywords: reliability, underwater, monitoring, sensor, pipeline.

\section{INTRODUCTION}

The continuous progress in communications and integrated systems has led to significant development in the field of industrial tasks and greater control. The great scientific advances in information and communication technology, the improvement of microelectromechanical systems, industrial intelligence, and process control have improved all areas of industrial practices related to oil processing. Underwater acoustic wireless sensor networks (UAWSN) are used in different applications such as data collection, coastal monitoring, earthquake forecasting, oil pipeline monitoring, and pollution detection. The harsh underwater environments impose various constraints, most notably high bit error, propagation delay, low bandwidth, and short-range communications [1]. All of these limitations reduce the performance and lifetime of sensor nodes, therefore require the deployment of nodes in different ways. Deployment strategies play an important role in the success of underwater sensor networks because they need safeguards to address key objectives such as improving network life and full connectivity [2]. In addition, deployment strategies should address the key factors associated with the node or network, such as delay, throughput, packet reception rate, transmission and reception capacity, and energy consumption [3]. The linearity of oil pipelines requires the establishment of a linear sensor network along the pipeline for monitoring. Underwater linear networks characterized by limited routing protocols due to the linearity of the network thus, the simple algorithm is required to reduce energy consumption [4].

The Smart Redirect or Jump Algorithm (SRJ) is an integration of two algorithms, Jump Always (JA) and Redirect Always (RA) algorithms [5]. The Advantages of the SRJ algorithm are through combining the jumping and redirect strategies in terms of the SRJ algorithm provides the ability to overcome failure nodes. This algorithm depends on the number of hops to determine the direction of packets to reduce the energy needed to send packets. The problem in this algorithm is the data relay nodes which are deployed around oil pipeline suffer from time and energy consumed during the process of relay data to the neighbor nodes which lead to result network delay, and the process of increasing transmission range of data relay nodes in opposite direction to be twice in case of no 
neighbor node available in default direction[6],[7].In addition, the sensors connected to the relay node may be out of network due to the failure of these nodes. This work proposes a new hierarchical network model by adding a new layer of nodes in the network model with improvements to the SRJ algorithm to increase the reliability of wireless sensor networks in an underwater environment.

\section{BACKGROUND AND RELATED WORK}

In previous years, Numerous research studies were conducted on non-linear sensor networks [8], [9], [10] and on linear sensor networks [11], [12], [13].several solutions have been proposed to address the factors imposed by the harsh underwater environment in terms of routing algorithms, network topology, and method of deployment of nodes depending on the type of these nodes with a short or long-range. The communication between nodes is limited as a result of the harsh underwater environment, and due to these factors in an underwater environment. The nodes suffer from failure which leads to the partial or total malfunction of the network, especially if these nodes are close to the sink as shown in Figure (1).

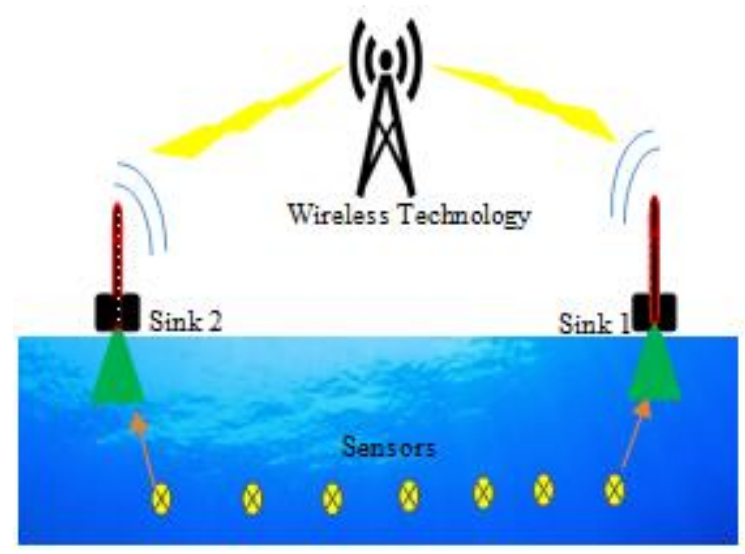

Figure1. Underwater linear wireless sensor network [14]

In [14 ],The enhanced algorithm for the deployment of a linear underwater wireless sensor network (EULWSND) is presented for improved durability in the collection of underwater linear sensor data deployment strategies were discussed and compared with the proposed strategy taking into consideration the linearity of the underwater pipeline and the heterogeneity of sensor nodes. The work presented in [5], Provides a comprehensive analysis of the integration of wiring with radio and acoustic signals to establish a reliable network, meet underwater challenges, compare network connectivity, network power supply continuity, and physical network security. The jumping or redirect protocol (SRJ) is proposed and compared with the previous methods to increase the reliability of the underwater sensor network. The author in [6], proposes increasing the transmission range for relay nodes in an adaptive manner called Adaptive Smart Redirect or Jump algorithm (ASRJ) depending on the status of the neighboring relay nodes in both directions to reduce the energy consumed in these nodes despite the increased network delay due to the increase in the number of hops.

\section{ENERGY CONSUMPTION AND END-TO-END DELAY}

The process of energy consumption of the underwater acoustic sensor networks (UASNs) depends on their placement and trade-off with the end to end delay. For the nodes system, a sufficient number of underwater nodes should be deployed to fully cover the oil pipeline because these nodes are connected by acoustic waves and all these nodes are subject to the same environmental standards [15].

The source signal level SSL which calculated during send packet from the source node to the gateway can be represented by equation (3) [16].

$S S L=A N L+S N R+T L-D I$

Where $A N L$ is an ambient noise level, $S N R$ represents the signal-to-noise ratio, $T L$ represents the transmission loss, and $D I$ represents the directivity index.

The transmission loss can be calculated by equation (2) [17].

$T L=10 \log d+A C d 10^{3}+T A$

International Journal of Innovative Research in Electronics and Communications 
Where $d$ represents the distance between the sender and the receiver, $A C$ represents the absorption coefficient, and $T A$ represents the transmission anomaly [18].

$S S L=10 \log (\operatorname{SIT} / 1 \mu \mathrm{Pa})$

Where SIT represents the intensity of the underwater signal in $\mu \mathrm{Pa}$ and can be expressed using equation (4).

For the transmission power, TP can be represented by equation (5)[19],[20].

SIT $=10^{\text {ss } / 10} \times 0.67 \times 10^{-18}$

$T P=2 \pi \times d \times h \times S I T$

Where SIT represents the intensity of an underwater signal, and $h$ represents the water depth in $\mathrm{m}$.

In UASN, the end-to-end delay (EED) can be calculated using equation (6) [20 ],[21].

$E E D=N\left(t_{p r o p(i, i+1)}+t_{p k t}\right)$

Where $t_{\text {prop }(i, i+l)}$ represent propagation time between sensor nodes $I$ and $i+l$ in underwater, $\mathrm{t}_{\mathrm{pkt}}$ represent time to transmit a data packet, and $\mathrm{N}$ is the number of hops.

\section{SMART REDIRECT OR JUMP Algorithm (SRJ)}

A-parent/child relationship

A hierarchical network model of the linear structure sensor network consists of three types of heterogeneous nodes [5], [6]. Each node has its functionality represented by data collection, routing, and dissemination to the control network center (NCC). Figure (2) shows heterogeneous nodes in the parent/child relationship, which represent the relationship among these nodes

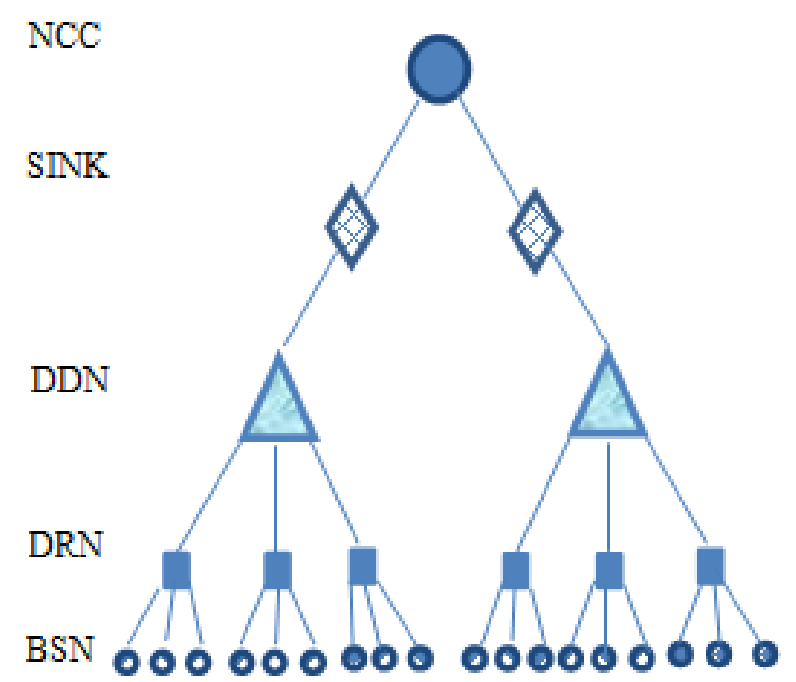

Figure2. .Parent/child relationship for the Smart Redirect or Jump algorithm [5]

\section{- Basic Sensing Nodes (BSNs)}

This node is one of the common nodes that are deployed along the oil pipeline to detect the corroding and leakage that occurs in the pipeline. These sensors send data to the relay node in the next layer of the network structure.

\section{- Data Relay Nodes (DRNs)}

The role of these nodes is to collect data from the (BSNs) then select the shortest route and forward data to the DDN to reduce the energy consumption

\section{- Data Dissemination Nodes (DDNs)}

These nodes collect data and direct to the sink which sends this data to Network Control Center (NCC), therefore this node has a higher communication capability. Based on DDNs deployment, it possible to divided linear networks into multiple segments. 


\section{B-MessagesRelaying by (DRN)}

Relay nodes forward data based on (jump or redirect strategies) therefore, each node has a primary parent (DDN1) and secondary parent (DDN2). BSNs send a packet to the relay node (DRN) where this node forward data to its neighbor in the default direction. If the neighbor node is not active, the jump strategy is used to reach the other nodes rely on jump factors (single or multi hope). In the case of, the jump strategy is failed, the redirect strategy is used. Each relay node contains the information of the neighboring nodes and the operational status of each node.

The direction of sending packets depends on the energy-consumption (Ex) in both directions [4]. If Ex1p < Ex2p, the packet direction is towards to the primary parent (DDN1), by contrast, the direction of the packet will be towards the secondary parent(DDN2).Figure (3) represents the direction of the packet based on energy-consumption. The energy-consuming in both directions based on the number of hops in each direction thus, each node must calculate the energy consumed to choose the shorter route, therefore, increase packets that are delivered to the parent node.

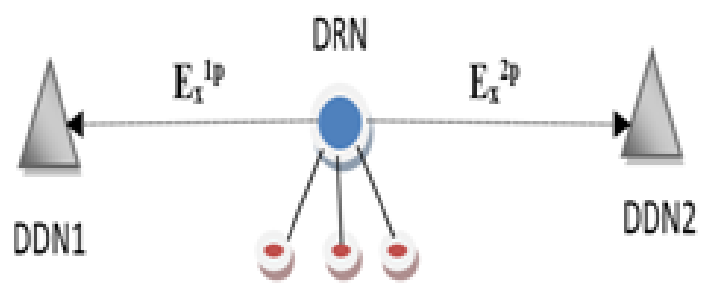

\section{BSNs}

Figure3. Packets direction based on number of hops [6]

\section{Proposed Methodology}

The deployment of nodes in underwater networks rely on the way to ensure communication between them. In the proposed approach, we add new nodes communicated with the sensors instead of the previous strategy where sensors communicated with the relay nodes directly. These nodes collect data and send it to the relay nodes depends on the status of these nodes, not on the number of hops in both directions. This node is called Aggregation Relay Node (ARN). This node does not transmit other network data, as in relay nodes, but only data from the communicated sensors. The new hierarchical network model is shown in Figure (5).

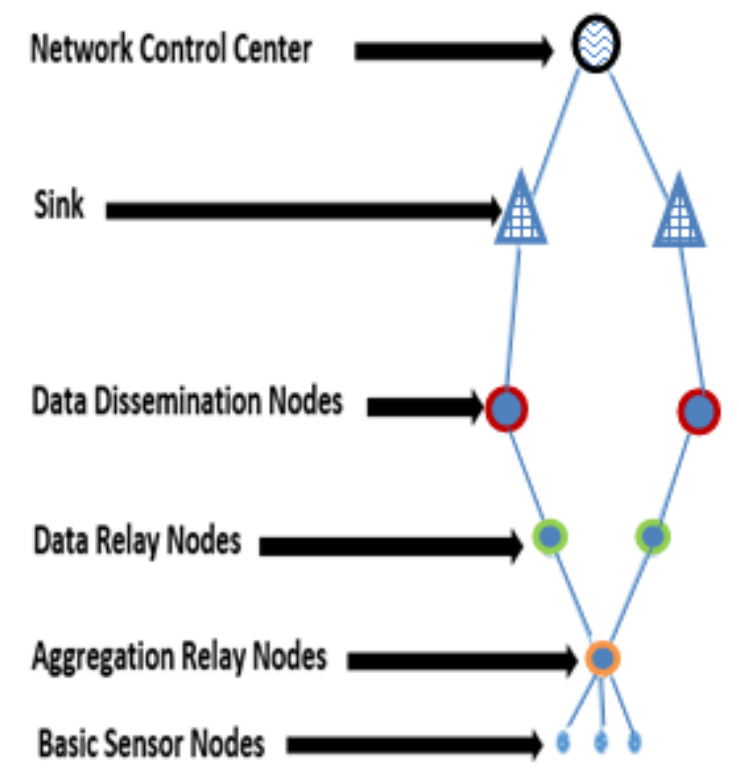

Figure4. Hierarchical representation of the new network model

The new structure is based on the proposed algorithm applied in (ARN). This algorithm called improved SRJ algorithm (ISRJ) which relies on the transmission of packets to the active nodes within the transmission range in the default direction. If a node fails, the packets are sent to the active node 
within the transmission range in the opposite direction. In case of failure to reach both directions, the transmission range is increased, to reach relay nodes. In the case of, there are no relay nodes, the packet is dropped. The routing of data received from the sensors by the ARN nodes reduces overwork on relay nodes and thus, saves the energy consumed with an acceptable end-to-end delay.

In the new hierarchical network model, the failure of the relay node does not necessarily mean that the sensors are out of service because the (ARN) node is communicated to other relay nodes. Also, the sensors do not need to search for a new node to connect to the data transfer as it is in the old structure in case of failure of the relay node and thus save energy consumed. Algorithm (1) represents the improved SRJ algorithm implemented in (ARN) node.

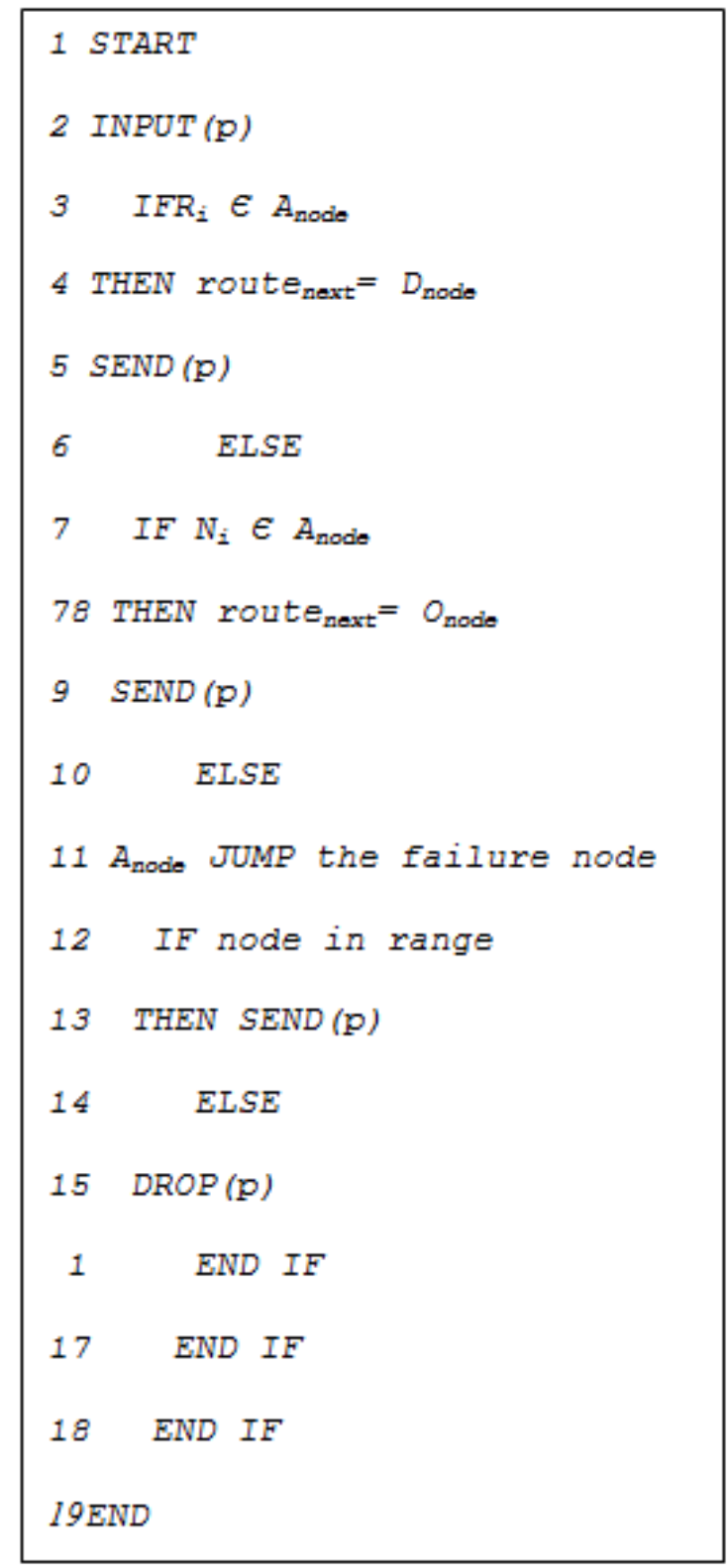

Algorithm1. ImprovementSRJ algorithm for ARNs

Depending on the state of the relay nodes on both sides of the ARN node, the packets are routed and the change of direction is prioritized before the jumping process in order to reduce the energy consumed.

\section{Simulation SCEnARios}

In this section, the proposed network model is simulated with the proposed algorithm to monitor the oil pipeline using two software tools $\mathrm{OMNeT}++$ and Matlab. The programming in the OMNeT++ simulator is dependent on the $\mathrm{C} / \mathrm{C}++$ language and NED (NEtwork Description). Network and link layers are protocol layers simulated using OMNeT ++.The third protocol layer is the physical layer 
which is simulated as a Mat lab function Integrated into OMNeT++. The physical layer can be simulated as a modulator, a channel, and a demodulator that developed by Borrowski [22].

Table1. Represent the simulation parameters

\begin{tabular}{|c|c|}
\hline Parameters & Value \\
\hline Pipeline length & $1000 \mathrm{~m}$ \\
\hline Type of nodes & BSN,ARN,DRN,DDN \\
\hline Total Number of DDN & 2 (one node every 1000meter) \\
\hline Total Number of DRN & 10 (one node every 80 meter \\
\hline Total Number of ARN & 5 (one node every 160 meters) \\
\hline $\begin{array}{c}\text { Total Number of BSN } \\
\text { per ARN }\end{array}$ & 4 (one node every 40 meters) \\
\hline Ranges of nodes & $50,100,120 \mathrm{~m}$ \\
\hline Bandwidth & $20 \mathrm{Khz}$ \\
\hline Packet size & 64 byte \\
\hline Hello Packet & 12 byte \\
\hline Propagation model & Underwater propagation \\
\hline Number of Sinks & 2 \\
\hline simulation time & $1000 \mathrm{~s}$ \\
\hline
\end{tabular}

Figure (5) represents the four-level hierarchical sensor network thathas adopted in this work.

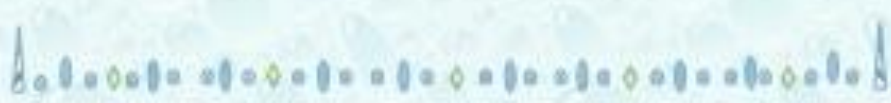

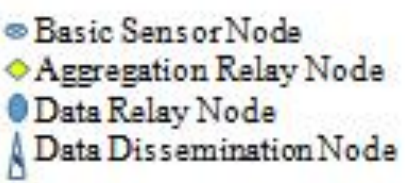

Fig5. New hierarchical sensor network

In figure (5), the heterogeneous nodes around the oil pipeline are distributed by estimating the transmission ranges of these nodes. BSN devices act as sensors that send their data to the ARN node, which in turn sends them to the relay node depending on the proposed algorithm. The relay nodes deliver the packets to the DDN nodes, which in turn send them to the sink that converts them to the network control center (NCC).

\section{RESULT}

The results in this work are compared against the old hierarchical network model with (SRJ) algorithm. The results are presented in the following figures.

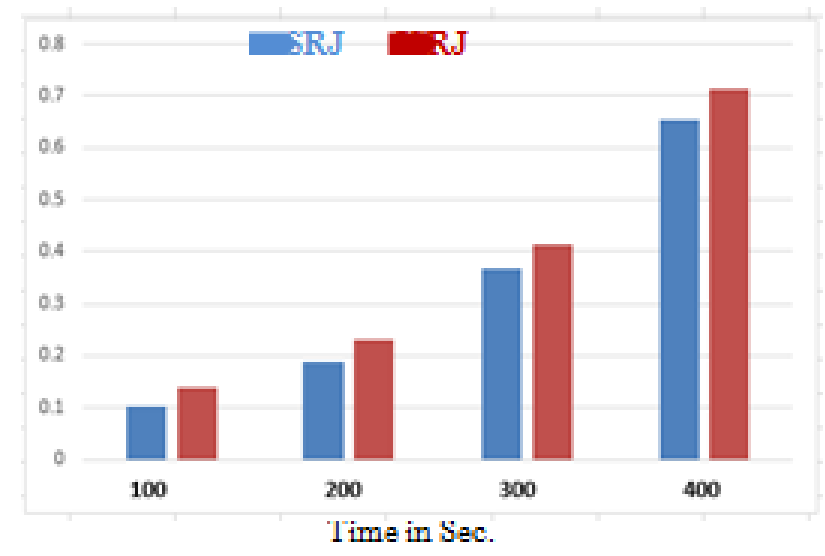

Figure6. Received Throughput 


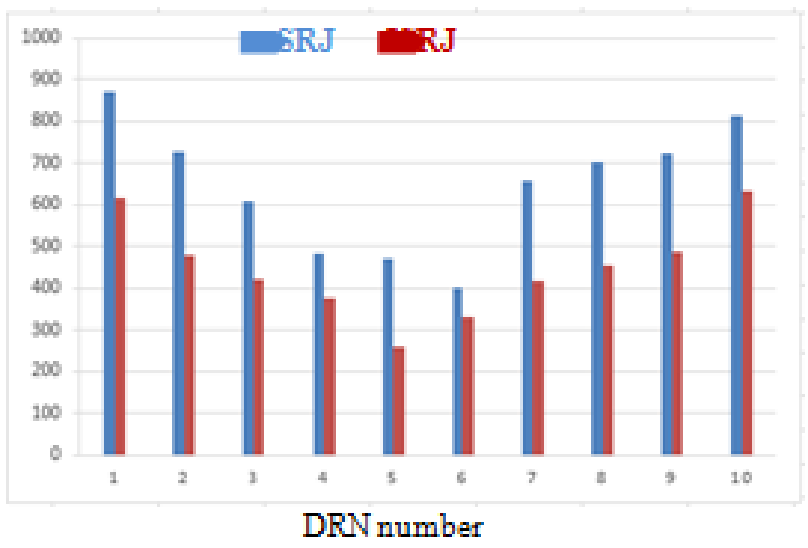

Figure7. Energy Consumption

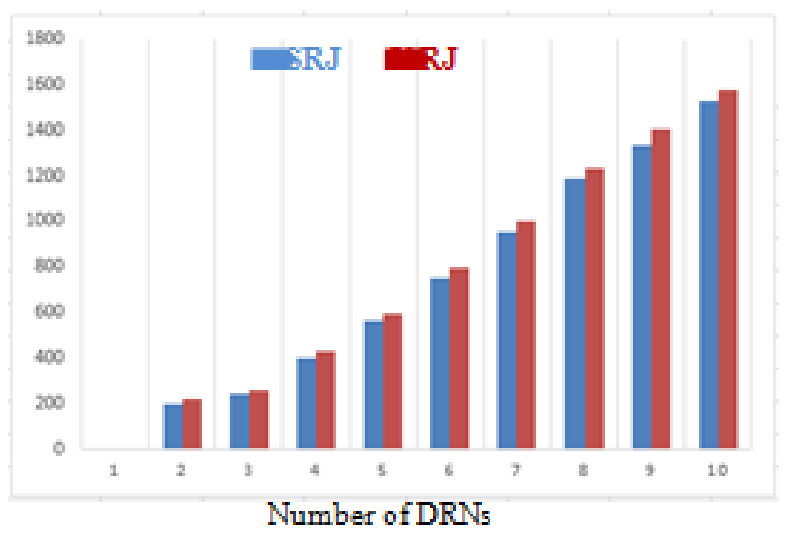

Figure8. End - to - End delay

Figure (6) represents the received throughput in different period's time. The process of adding the ARN node leads to improve the performance of the network in terms of increasing the probability of the arrival of packets sent by the sensors. It also reduces the delay between relay nodes due to reduced overwork. Also, the proposed model provides more productivity in case of relay nodes fail because ARN node will provide another path for the sensors towards the relay nodes. The difference between the two protocols is mainly due to the fact that ISRJ applied in ARN works faster and more efficiently during the setup and communication process, especially in case of transmission to the farther relay node with its transmission range.

The process of energy consumption of the UWSNs depends on the placement of the nodes and tradeoff with the end to end delay. For the system of nodes, a sufficient number of underwater nodes should be deployed to fully cover the oil pipeline because these nodes are connected by acoustic waves and all these nodes are subject to the same environmental standards. In this experiment, better results were obtained after using the proposed new network hierarchy. In Figure (7), the new structure with the proposed ISRJ algorithm outperforms the old structure using the SRJ algorithm due to the increase in the number of nodes and the reduction of overwork on the relay nodes by deciding to route packets by the ARN node and thus reducing the memory used for storage. In addition, the ARN node increases the transmission range when there are no active relay nodes in directions, extending network life and increasing reliability.

Figure (8) shows the end - to - end delay of the two compared strategies. The experience of the new hierarchy shows that end-to-end delays are acceptable compared to the SRJ algorithm. The ARNs that are added to the network increase the number of hops by one hop for the transmitted data because these nodes do not participate in the transmission of other data in the network. The number of jumps range affects the delay from source to destination, which should be a trade-off between energy consumption and delay from start to finish. In this work, the ARN node sends data to the farthest relay node within its scope to minimize the delay caused by increasing the number of hops. The processes in the relay nodes are better because of the new structure with the proposed algorithm, which reduced the overwork on these nodes 


\section{CONCLUSION}

The process of monitoring the underwater oil pipelines receives wide attention due to the importance of these pipelines for the transfer of crude oil. Added nodes are used to collect data from the sensors and directed to the relay nodes and propose an algorithm based on the status of the nodes in both directions. The results show the superiority of the new network hierarchy. In the case of the first scenario, the received network throughput between the two strategies was compared based on different period's time. The ISRJ approach outperforms in terms of packet delivery ratio over SRJ due to maintaining the number of sensor nodes in case any relay node fails in the proposed strategy as the nodes are directly connected to ARNs that provide greater network reliability. The end-to-end delay in the first simulation scenario is acceptable when compared to the amount of energy consumed in the ISRJ strategy. The use of ARNs in the network led to reducing the energy consumed in the relay nodes, resulting in a longer lifetime of these nodes, thus increased network reliability. The trade-off between end-to-end delay and energy consumption has shown that the network is not much affected by the end-to-end delay and the energy consumed in the ISRJ approach is lower than in SRJ.

\section{REFERENCES}

[1] M. Barbeau, S. Blouin, G. Cervera, J. Garcia-Alfaro, B. Hasannezhad, and E. Kranakis, "Simulation of Underwater Communications with Colored Noise Approximation and Mobility," p. 6.

[2] G. Heredia, "Multi-Unmanned Aerial Vehicle (UAV) Cooperative Fault Detection Employing Differential Global Positioning (DGPS), Inertial and Vision Sensors," p. 15, 2009.

[3] Premalatha, J., and PM Joe Prathap. "A Survey on Underwater Wireless Sensor Networks: Progresses, Applications, and Challenges." MATEC Web of Conferences, Taiwan, May, 2016.

[4] Faheem et al., "QoSRP: A Cross-layer QoS Channel-Aware Routing Protocol for the Internet of Underwater Acoustic Sensor Networks," Sensors, vol. 19, no. 21, p. 4762, Nov. 2019, doi: $10.3390 / \mathrm{s} 19214762$.

[5] N. Mohamed, I. Jawhar, J. Al-Jaroodi, and L. Zhang, "Sensor Network Architectures for Monitoring Underwater Pipelines," Sensors, vol. 11, no. 11, pp. 10738-10764, Nov. 2011.

[6] E. F. Farid, A. A. Mahmoud, and A. M. Bahaa-Eldin, "Adaptive Smart Redirect and Jump Algorithm (ASRJ) for Wireless Sensor Networks," in 2015 Tenth International Conference on Computer Engineering \& Systems (ICCES), Cairo, Egypt, 2015, pp. 247-252

[7] M. Z. Abbas, K. Abu Bakar, M. Ayaz Arshad, M. Tayyab, and M. H. Mohamed, "Scalable Nodes Deployment Algorithm for the Monitoring of Underwater Pipeline," TELKOMNIKA, vol. 14, no. 3, p. 1183, Sep. 2016.

[8] M. S. Choi, W. Yaqiong, K. T. Kim, S. J. Yong, andH. Y. Youn, "A New Polling Protocol with EfficientCluster Creation for WSN," in 2010 IEEE/ACMInt'l Conference on Green Computing and Communications \& Int'l Conference on Cyber,Physical and Social Computing, 2010, pp. 357-362.

[9] K. Almi'ani, A. Viglas, and L. Libman, "MobileElement Path Planning for Time-Constrained DataGathering in Wireless Sensor Networks," in 201024th IEEE International Conference on Advanced Information Networking and Applications, 2010, pp.

[10] P. N. E. S. Barbosa, N. M. White, and N. R. Harris, "Wireless Sensor Network for Localized Maritime

[11] Monitoring," in 22nd International Conference onAdvanced Information Networking and ApplicationsWorkshops (aina workshops 2008), 2008, pp. 681-

[12] M. Zimmerling, W. Dargie, and J. Reason,"Localized power-aware routing in linear wirelesssensor networks," in CASEMANS, 2008.

[13] M. Zimmerling, "Energy-efficient routing in linearwireless sensor networks," in Mobile Adhoc and Sensor Systems, 2007..

[14] Z. Guo, H. Luo, F. Hong, and P. Zhou, "GRE:Graded Residual Energy Based Lifetime Prolonging Algorithm for Pipeline Monitoring Sensor," in 2008 Ninth International Conference on Parallel and Distributed Computing, Applications and Technologies, 2008, pp. 203-210.

[15] Z. Ahmed and K. Abu Bakar, "An Enhanced Underwater Linear Wireless Sensor Network Deployment Strategy for Data Collection," Int J Innov Comp, vol. 8, no. 3, Nov. 2018, doi: 10.11113/ijic.v8n3.195.

[16] 686.843-850.G. Tuna and R. Das, "Trade-off Analysis of Underwater Acoustic Sensor Networks," p. 7, 2017.

[17] Michailidis E T, Tuna G, Gezer G, Potirakis S M and Gulez K 2014 ANN-Based Control of aMulti-Boat Group for the Deployment of an Underwater Sensor Network International Journal of Distributed Sensor Networks 2014786154. 
[18] Stojanovic M and Preisig J 2009 Underwater acoustic communication channels: Propagation models and statistical characterization IEEE Communications Magazine 47(1) 84-9.

[19] Proakis J G 1995 Digital communications (3rd ed.) (New York: McGraw Hill).

[20] Urick R J 1983 Principles of Underwater Sound (Mcgraw-Hill).

[21] Felemban M and Felemban E 2013 Energy-delay tradeoffs for Underwater Acoustic Sensor Networks Proc. First International Black Sea Conference on Communications and Networking (Batumi, Georgia) pp. 45-9.

[22] Cui S, Madan R, Goldsmith A and Lall S 2005 Energy-delay tradeoffs for data collection intdma-based sensor networks Proc. IEEE International Conference on Communications (Seoul, Korea) 5 pp. 3278-84.

[23] B. S. Borowski, Application of channel estimation to underwater, acoustic communication.PhD thesis, Stevens Institute of Technology, Castle Point on Hudson, Hoboken NJ, 2010.

Citation: Wassim M.Jassim, A.E.Abdelkareem, "Improved the Monitoring Performance of Oil Pipeline Using Underwater Wireless Sensor Network”, International Journal of Innovative Research in Electronics and Communications, 7(1), pp. 28-36. DOI: http://dx.doi.org/10.20431/2349-4050.0701004

Copyright: (C) 2020 Authors, This is an open-access article distributed under the terms of the Creative Commons Attribution License, which permits unrestricted use, distribution, and reproduction in any medium, provided the original author and source are credited. 